Rev. Adm. Saúde (On-line), São Paulo, v. 20, n. 80: e246, jul. - set. 2020, Epub 30 set. 2020 http://dx.doi.org/10.23973/ras.80.246

RELATO DE CASO

\title{
Concepção e divulgação de um e-survey para pesquisa em administração aplicada em enfermagem
}

Design and divulgation of an e-survey for research in nursing administration

\section{Quevellin Alves dos Santos Francisco', Maria Regina Martinez ${ }^{2}$}

1. Enfermeira, mestre em enfermagem. Chefe de enfermagem do Instituto Claudia Marques de Pesquisa e Desenvolvimento, Pouso Alegre MG.

2. Enfermeira, doutora em enfermagem. Professora do Curso de Medicina da Universidade Federal de Alfenas, Alfenas MG.

\section{RESUMO}

Análise do processo de concepção e divulgação de um e-survey desenvolvido como instrumento de coleta de dados para um estudo quantitativo, descritivo, de corte transversal, do tipo survey, que resultou na dissertação de mestrado "Participação dos gestores de enfermagem na gestão de pessoas: um estudo do tipo survey". As etapas da elaboração do e-survey foram: concepção, divulgação e envio do questionário através de uma rede social entre os anos de 2018 e 2019. As pesquisas on-line aproximam pesquisadores-participantes, porém garantem privacidade e comodidade. Para estas pesquisas é primordial a definição de um trajeto metodológico, pois os resultados obtidos podem ser usados para tomar decisões importantes no âmbito da prática da enfermagem.

Palavras-chave: Inquéritos e Questionários; Enfermagem; Serviços On-Line; Pesquisa em Enfermagem; Internet

\section{ABSTRACT}


Process analysis the conception and dissemination of an e-survey developed as a data collection instrument of a quantitative, descriptive, cross-sectional Survey study, which resulted in the master dissertation "Participation of nursing managers in management. of people: a Survey study". The stages of the preparation of the e-survey were conception, dissemination and sending of the questionnaire through a social network between 2018 and 2019. Online surveys bring research participants closer together but guarantee privacy and convenience. For this research it is essential to define a methodological path because the results can be used to make important decisions in the scope of nursing practice.

Keywords: Surveys and Questionnaires; Nursing; Online services; Nursing research; Internet

\section{INTRODUÇÃO}

As pesquisas do tipo survey (ou levantamento de campo) se caracterizam pela interrogação direta das pessoas sobre um determinado comportamento, atitude ou situação de vida que as envolve, normalmente utilizando um único instrumento de coleta de dados, o questionário. Basicamente, solicitam-se informações a um grupo significativo de pessoas acerca do problema estudado e, após a organização e análise quantitativa dos dados obtidos, são tecidas as conclusões pertinentes (1).

A aplicação de questionários nas pesquisas do tipo survey por meio de ferramentas disponíveis na internet reduz o custo envolvido na pesquisa, possibilita a ampliação da amostragem e do acesso a pessoas em áreas mais distantes, reduz o tempo utilizado para coleta de dados e aumenta a efetividade na compilação de grandes quantidades de dados(2).

Em meados de 2019, mais de 4.4 bilhões de pessoas ao redor do mundo foram consideradas usuárias de internet, sendo que, no Brasil, mais de $70 \%$ da população utiliza a internet (3). Portanto, pesquisas do tipo survey aplicadas on-line tem o potencial de fornecer dados representativos que permitam a obtenção de inferências válidas.

Além das vantagens envolvidas na condução desse tipo de pesquisa para o pesquisador, responder questionários disponíveis on-line tende a ser mais conveniente para o participante da pesquisa. Suas respostas podem ser geradas em momentos considerados mais convenientes, independentemente da disponibilidade do pesquisador, temas constrangedores podem ser abordados sem a sua exposição direta e os questionários podem ser completados de acordo com sua preferência (4). 
Há várias ferramentas on-line disponíveis para a aplicação de questionários de pesquisas do tipo survey. O serviço oferecido pelo site SurveyMonkey (https://pt.surveymonkey.com/) mostra-se especialmente vantajoso para pesquisadores brasileiros por oferecer uma plataforma em português e funcionalidades gratuitas ou com baixo custo. Dentre as funcionalidades desta plataforma destacam-se: (i) pesquisas personalizáveis gratuitas, (ii) análise de dados, (iii) seleção de amostras, (iv) ferramentas de representação de dados $(1,4)$.

Os profissionais da enfermagem podem se beneficiar da pesquisa on-line para obtenção de dados, para tanto, além de habilidades gráficas de elaboração das pesquisas, é primordial que o pesquisador tenha o conhecimento adequado para a condução de uma pesquisa metodologicamente segura.

Objetivo do trabalho: analisar o processo de concepção e divulgação de um esurvey para aquisição de dados para um estudo realizado no âmbito de um Programa de Pós-graduação em Enfermagem na Universidade Federal de Alfenas, como parte de uma dissertação de mestrado.

\section{MÉTODOS}

O estudo foi realizado no âmbito do Programa de Pós-graduação em Enfermagem da Universidade Federal de Alfenas, como parte de uma dissertação de mestrado. Tratou-se de um estudo quantitativo, descritivo, de corte transversal, do tipo survey que resultou na dissertação de mestrado "Participação dos gestores de enfermagem na gestão de pessoas: um estudo do tipo survey".

O objetivo do estudo foi analisar a participação dos gestores de enfermagem nos processos de gestão de pessoas, conhecer o perfil dos gestores de enfermagem no cenário brasileiro e descrever as ferramentas de gestão de pessoas utilizadas por gerentes de enfermagem.

Neste estudo o tamanho da amostra foi definido considerando-se o nível de precisão desejado, em intervalos de $95 \%$ de confiança e $5 \%$ de erro amostral, sendo, então, estabelecida uma amostra de 385 enfermeiros ou enfermeiras atuantes como gestores de enfermagem em estabelecimentos de saúde no Brasil. A aproximação com estes gestores foi feita por meio de uma rede social profissional (Linkedln) e do envio de uma mensagem de texto contendo o link de acesso ao questionário.

O projeto de pesquisa foi aprovado pelo Comitê de Ética em Pesquisa da Universidade Federal de Alfenas, pelo no de CAAE: 91351518.9.0000.5142 e no de parecer: 2.773 .753 (17 de julho de 2018). Para participar da coleta de dados por meio do preenchimento do questionário virtual, o sujeito de pesquisa tomava conhecimento e autorizava a utilização dos dados fornecidos com o acesso e aceite do Termo de Consentimento Livre e Esclarecido (TCLE). 


\section{RESULTADOS}

\section{Concepção do questionário}

Foi desenvolvido um questionário virtual (e-survey) contendo 22 questões, sendo 21 de múltipla escolha e uma com escala de mensuração tipo Likert. As questões foram organizadas e sequenciadas de forma a atender três critérios: (i) sequência lógica dos assuntos de forma a facilitar o raciocínio e o preenchimento correto das questões; (ii) inter-relacionamento entre as questões; (iii) evolução da resposta ao questionário, considerando as divisões de páginas e o tempo necessário para o preenchimento de cada uma com o intuito de diminuir a possibilidade de abandono antes da conclusão do preenchimento.

O questionário foi disponibilizado na Internet por meio de uma plataforma de pesquisa on-line com vistas a possibilitar o acesso e participação de indivíduos de todas as regiões do Brasil. A plataforma escolhida foi a SurveyMonkey.

A SurveyMonkey, trata-se de uma companhia baseada em nuvem, de desenvolvimento de pesquisas on-line, fundada em 1999 por Ryan Finley. Provê pesquisas personalizáveis gratuitas, bem como uma suíte de programas que inclui análise de dados, seleção de amostras, eliminação de vieses, e ferramentas de representação de dados (1).

Anteriormente ao envio do questionário para os participantes da pesquisa, um teste piloto foi aplicado para identificar elementos como fidedignidade, validade e operatividade do e-survey. Não sendo encontradas divergências entre as repostas, as questões foram mantidas e os participantes do teste piloto foram incluídos na amostra final do estudo. O questionário completo pode ser acessado através do link https://pt.surveymonkey.com/r/YGLH3KF.

O período de coleta de dados foi entre os anos de 2018 e 2019 e foram necessários 10 meses para o recebimento de 414 questionários completamente preenchidos. Os custos para a concepção do questionário resumiram-se no pagamento da taxa de hospedagem e confecção do questionário na plataforma on-line, calculados em torno de $R \$ 90,00$ reais por mês.

\section{Divulgação e envio do questionário}

A aproximação com a população alvo aconteceu através da rede social Linkedln ${ }^{\mathrm{TM}}$. Para a divulgação do estudo, foram postados links em fóruns e páginas com participação do público alvo na rede social. O LinkedlnTM firmase como uma das plataformas digitais mais relevantes da atualidade. Dos usuários brasileiros, dois em cada três têm diploma universitário e três em quatro usuários consideram o LinkedlnTM uma fonte confiável de informações de indústrias e de empresas. No Brasil são mais de 21 milhões de usuários, sendo o terceiro país em quantidade de perfis registrados. Como a rede social 
profissional mais usada no mundo, o LinkedInTM é um ambiente profícuo para negócios globais, regionais e locais(5).

Os participantes da pesquisa puderam indicar ou encaminhar a pesquisa para pessoas com características de interesse para a pesquisa. Neste caso, foram alcançados indivíduos qualificados para responder ao questionário, mas que não poderiam ser acessados somente com a técnica de amostragem estabelecida.

Nesse sentido, o estudo contou com a divulgação do Conselho Federal de Enfermagem (COFEN) e dos Conselhos Regionais de Enfermagem (COREN), que postaram em suas páginas nas redes sociais reportagens divulgando a realização a pesquisa (http://www.cofen.gov.br/pesquisa-avalia-estrategias-degestao-de-pessoas-aplicadas-a-enfermagem_66493.html).

O e-survey foi encaminhado a 920 perfis de gestores de enfermagem na rede social LinkedIn ${ }^{\mathrm{TM}}$ e obteve uma taxa de resposta de $45 \%$. A taxa de conclusão do questionário foi de $100 \%$ e o tempo médio para resposta foi de 7 minutos e 10.segundos $\mathrm{Na}$ verificação individual das respostas de cada respondente foi apresentado o endereço de Internet Protocol (IP) do computador, com o qual foi aplicado o questionário e, ainda, data e hora inicial e final em que foi respondido. Foi permitido somente o preenchimento de um questionário por endereço de IP, essa medida foi tomada visando impedir a duplicidade de questionários respondidos por um mesmo participante.

\section{Análise e apresentação dos resultados}

Os dados foram importados e analisados diretamente pelo Microsoft Power BI Desktop versão 2.68.5432.661 64-bits (abril de 2019), fato que dispensou a dupla digitação, uma vez que esses dados não foram manipulados manualmente.

Os dados foram importados da plataforma de pesquisa e-survey para o Microsoft Power BI como arquivos .xls para análise estatística descritiva. A apresentação dos dados foi feita por tabela de frequência e figuras, além de painéis de controle interativos. A Figura 1 ilustra as etapas descritas: concepção do questionário, divulgação e envio do questionário, análise e apresentação dos resultados. 


\section{CONCEPÇÃO DO QUESTIONÁRIO}

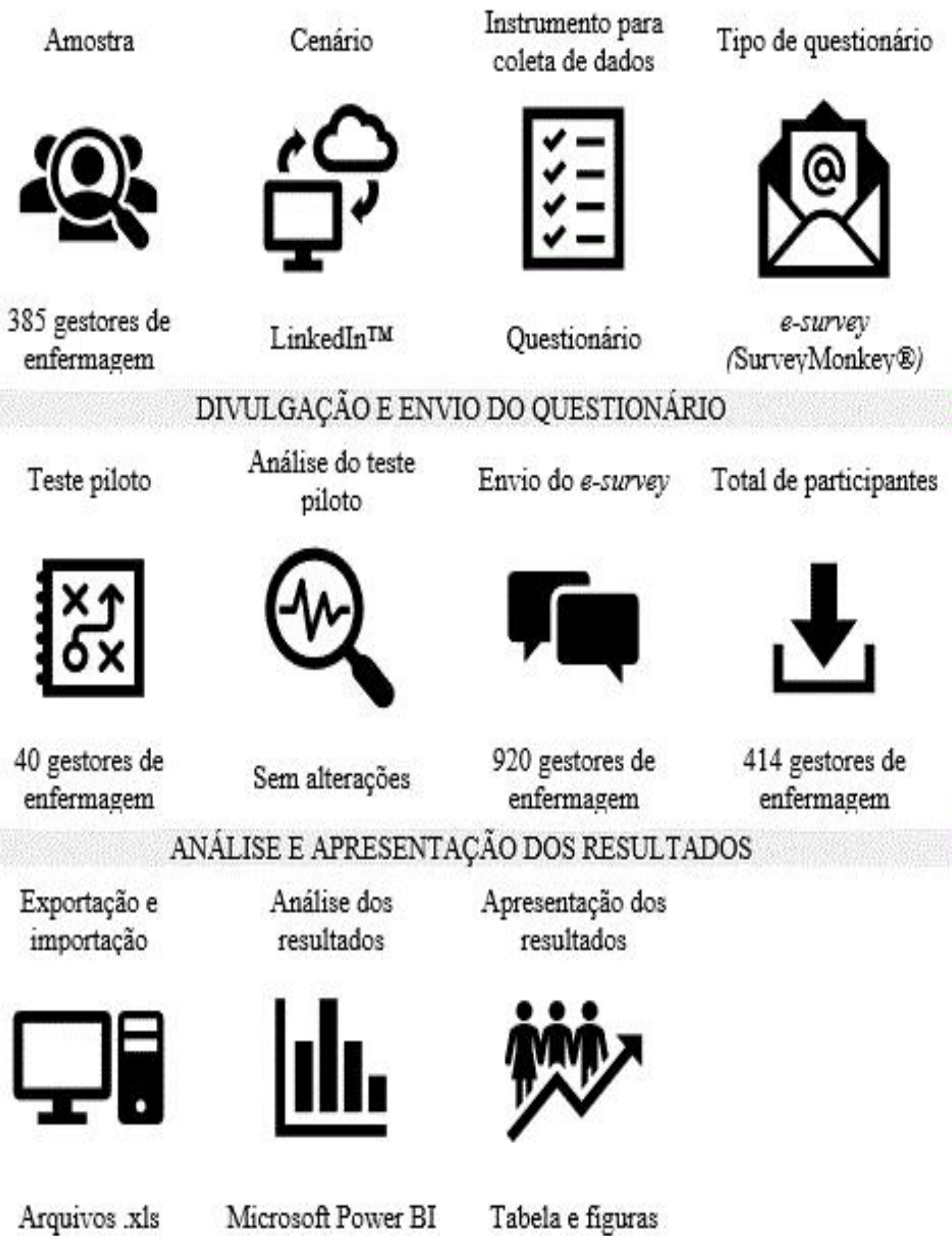

Figura 1. Etapas do desenvolvimento do e-survey. 
A Internet intensifica os canais de participação do cidadão. A utilização de $e-$ surveys para pesquisas permite a aproximação com pessoas de diferentes regiões e espaços na sociedade. Entretanto, ainda precisam ser discutidas questões como acessibilidade e conscientização, que por sua vez requerem não apenas o conhecimento para operar os recursos eletrônicos, mas, sobretudo, a consciência da importância do papel dos participantes nestas pesquisas (6).

Este tipo de pesquisa oferece vantagens em relação aos questionários tradicionais. Entre as vantagens estão a otimização do tempo, o baixo custo e a redução de erros com a entrada de dados manualmente. Os e-surveys simplificam o processo de coleta de dados, formatam e inserem as respostas diretamente em um banco de dados para análise. Estes são customizáveis e passíveis de verificação e correção em tempo real, aumentando a precisão do processo de coleta. Algumas plataformas permitem a formatação com layouts atraentes e fáceis de ler, o que melhora a taxa de resposta (7).

Outra vantagem é o acesso ser por meio de endereços eletrônicos que podem ser inseridos no corpo de um e-mail convite explicando a finalidade do questionário e informações de autoria. Além do que, atualmente, recursos cada vez mais inovadores estão sendo utilizados, como o preenchimento dos questionários em modo off-line, envio através de redes sociais, e aplicativos para preenchimento em dispositivos portáteis (mobiles) $(6,8)$.

Além das vantagens mencionadas acima, os questionários on-line estão sendo utilizados para pesquisas de opinião de mercado através de modelos de crowdsourcing, que conta com a mão de obra e conhecimentos coletivos para a criação ou desenvolvimento de produtos. Neste sentido, o Amazon Mechanical Turk aplica-se quando é desejável obter informações rapidamente sobre comportamentos e opiniões de usuários. Assim como o SurveyMonkey, o Amazon Mechanical Turk apresenta resultados precisos e confiáveis (11).

Outra estratégia que tem sido utilizada para gerar o engajamento no preenchimento dos questionários é a gamificação. Muito embora não tenha sido aplicada na elaboração do presente estudo, tem sido utilizada em outros estudos de forma a melhorar a representatividade da amostra e reduzir o risco de viés de não resposta (9). Elementos utilizados nos jogos influenciam as reações dos indivíduos ao interagir com pesquisas gamificadas. A gamificação serve como um sinal positivo e aumenta as reações afetivas, e estes avanços têm implicações teóricas e práticas para melhorar o design de pesquisas online existentes (10).

\section{Limitações do estudo}

Destaca-se como limitação a dificuldade de acesso aos profissionais de algumas regiões. Por mais que este tipo de pesquisa tenha como facilitador 0 quesito de aproximação, profissionais de algumas regiões não retornaram o questionário enviado. 


\section{Contribuições para a área}

O presente relato contribui para reforçar a existência e as possibilidades da pesquisa on-line. Este tipo de pesquisa garante a participação de sujeitos de realidades distantes dos pesquisadores. Dados como os que foram pesquisados, talvez causassem desconforto se fossem questionados pessoalmente. A partir das respostas obtidas com o e-survey foi possível definir um perfil da gestão de enfermagem no cenário brasileiro e das ferramentas utilizadas para a gestão de pessoas.

\section{CONSIDERAÇÕES FINAIS}

As pesquisas on-line aproximam pesquisadores-participantes, porém garantem privacidade e comodidade. $\mathrm{O}$ deslocamento torna-se desnecessário, e a identificação do participante limita-se ao IP do computador utilizado para responder o questionário. Ressalva-se a importância de definição de um trajeto metodológico na elaboração do e-survey, pois quando bem elaborados, os resultados obtidos são concretos em relação a pergunta de pesquisa, e as informações podem ser usadas para tomar decisões importantes no âmbito da prática da enfermagem.

Conhecer o contexto para a aplicação do e-survey e a definição da estratégia de divulgação são grandes aliados para o sucesso da pesquisa, portanto é primordial a aplicação do teste piloto. $\mathrm{O}$ estudo das plataformas de hospedagem dos questionários deve ser realizado e a escolha vislumbrar o perfil da população estudada. Estratégias como o uso da gamificação e opções de resposta offline com posterior transmissão, são recursos que otimizam a taxa de resposta.

Sugere-se para estudos futuros a avaliação do conhecimento e a utilização dos profissionais da enfermagem dos questionários on-line (e-survey) em pesquisas acadêmicas.

\section{REFERÊNCIAS}

1. Formigoni Carvalho Walter OM. Análise de ferramentas gratuitas para condução de survey online. Prod Produção. 2013 Jul; 14(2).

2. Regmi PR, Waithaka E, Paudyal A, Simkhada P, Van Teijlingen E. Guide to the design and application of online questionnaire surveys. Nepal $\mathrm{J}$ Epidemiol. 2017 May; 6(4):640-4. 
3. World Internet Users Statistics and 2019 World Population Stats [Internet]. 2019 [cited 2019 Aug 6]. Disponível em:

https://www.internetworldstats.com/stats.htm

4. Wright KB. Researching Internet-Based Populations: Advantages and Disadvantages of Online Survey Research, Online Questionnaire Authoring Software Packages, and Web Survey Services. J Comput Commun. 2006 Jun; 10(3):00-00.

5. Sebrae. Série Canais digitais para pequenos negócios. 2015. Recuperado em 10 de dez. 2019 de https://www.sebrae.com.br/sites/PortalSebrae/ufs/ap/artigos/conheca-aserie-canais-digitais-para-pequenos-negocios.

6. Cope DG. Using Electronic Surveys in Nursing Research. Oncol Nurs Forum. 2014 Nov; 41(6):681-2.

7. Solomon DJ. Conducting Web-based surveys. Pract Assessment, Res Eval. 2001; 7(19):1-5.

8. Chung S-Y, Hacker ED, Rawl S, Ellis R, Bakas T, Jones J, et al. Using Facebook in Recruiting Kidney Transplant Recipients for a REDCap Study. West J Nurs Res. 2019 Mar; 019394591983260.

9. Blumenberg C, J D Barros A. Integrating REDCap to a gamified web-based platform used in a birth cohort follow-up: the coortesnaweb project. 2018.

10. Triantoro T, Gopal R, Benbunan-Fich R, Lang G. Would you like to play? A comparison of a gamified survey with a traditional online survey method. Int J Inf Manage. 2019; 49(April):242-52.

11. Bentley FR, Daskalova N, White B. Comparing the Reliability of Amazon Mechanical Turk and Survey Monkey to Traditional Market Research Surveys. In: Proceedings of the $2017 \mathrm{CHI}$ Conference Extended Abstracts on Human Factors in Computing Systems - CHI EA '17. New York, New York, USA: ACM Press; 2017. p. 1092-9.

Recebido: 24 de setembro de 2020. Aceito: 30 de setembro de 2020

Correspondência: Quevellin Alves dos Santos Francisco. E-mail: quevellinsantos@gmail.com

Conflito de Interesses: os autores declararam não haver conflito de interesses. 
(C) This is an Open Access article distributed under the terms of the Creative Commons Attribution License, which permits unrestricted use, distribution, and reproduction in any medium, provided the original work is properly cited 